\title{
MEASUREMENT BASED PERFORMANCE PREDICTION OF THE EUROPABRÜCKE AGAINST TRAFFIC LOADING
}

\author{
R. Veit, H. Wenzel \\ Vienna Consulting Engineers (VCE) Holding GmbH, Austria \\ E-mail: veit@vce.at, wenzel@vce.atＷebsite: vce.at
}

\begin{abstract}
Bridges are ageing and traffic is growing, which creates a demand for accurate fatigue life assessment. The Europabrücke - a well known Austrian steel bridge near Innsbruck, opened in 1963 - is one of the main alpine north-south routes for urban and freight traffic. A long-term preoccupation of VCE with BRIMOS $\AA$ (BRIdge MOnitoring System) on the Europabrücke (since 1997) and the assessed prevailing vibration intensities with regard to fatigue problems and possible damage led to the installation of a permanent measuring system in 2003.

A lot of investigation has already been devoted to reach the superior goal, which is the determination of the relation between the randomly induced traffic loading (vehicles per day) and the fatigue-relevant, dynamic response of the structure, exclusively caused by freight traffic. As life-time predictions in modern standards depend on lots of assumptions, the emphasis is to replace those premises - referring to loading - by measurements. In that context the present work is focused on three levels:
\end{abstract}

- Level I : Global behaviour in dependence of all relevant loading cases (based on laser-displacement measurement)

- Level II : Cross-sectional behaviour under special consideration of the cantilever regions (based on laser-calibrated acceleration measurement)

- Level III : Local systems analysing the interaction between tyres and the beam-slab connections (based on inductive displacement transducers)

In each of these levels of analysis the consumption of the structure's overall-capacity per year is to be determined. An indispensable requirement is to reduce the permanent monitoring system's data by Rainflow-Counting, describing the remaining fatigue-relevant recurring response-cycles in different categories of intensity and occurrence. As the present lifetime calculations are performed in terms of stresses by means of damage-accumulation, global and local Finite Element Analysis is necessary for the transition of measuring data. The detailed knowledge about the progression of the prevailing traffic from the very beginning up to these days and the implementation of published future trend studies for the next ten years can be used for an extrapolation of the measured impact for the whole lifetime. As this research work tries to encourage in-situ measurements instead of "design situations", it is also aspired to analyse the consequence of statistical scatter in each level of impact as well as for fatigue resistance.

Key words: Steel Bridge, Permanent Monitoring System, Pattern Recognition, Nominal \& Structural Stresses, Damage Accumulation, Statistical Scatter 


\section{Introduction}

Previous measurements at the Europabrücke matched very well with the comparative analytical calculations, but they also exhibited the remarkable loading impact. Currently the bridge is stressed by more than 30000 motor vehicles per day (approximately $20 \%$ freight traffic). The superstructure is represented by a steel box girder (width $=10 \mathrm{~m}$; variable height along the bridge-length $4,70-7,70 \mathrm{~m}$ ) and an orthotropic deck and bottom plate. This motorway bridge with six spans differing in their length (longest span 198m, supported by piers with an elevation of $190 \mathrm{~m}$ ) and a total length of $657 \mathrm{~m}$ comprises six lanes, three for each direction distributed on a width of almost 25 meters.

To reach the already defined goals, a permanent monitoring system has been developed in a stepwise manner (Figure 1). It consists of 24 measuring channels (sampling rate $100 \mathrm{~Hz}$ ) representing the main span's, the pier's and the cantilever's accelerations, the abutment's dilatation, wind speed and direction, and temperatures at several locations.

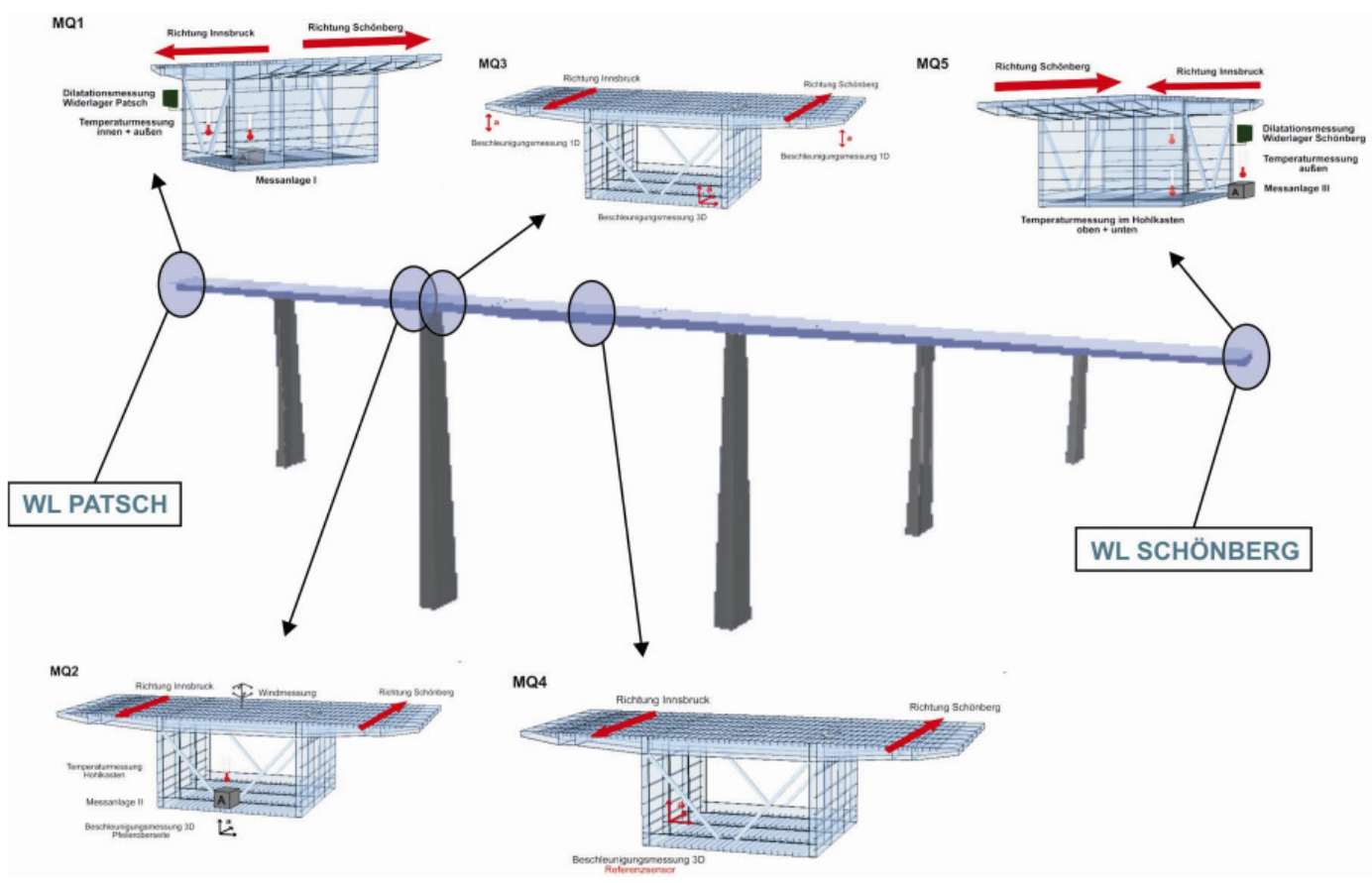

Fig. 1. - The permanent monitoring system and its several measurement sections

The research work discussed in the present paper started with the installation of the permanent monitoring system. An exhaustive explanation of the approach in general has already been given in [1]. The present contribution explicitly deals with measurement data and how they were made available and conditioned. Thus the main goal, the substitution of the standard's premises - referring to loading - has been reached in a quite innovative manner. 


\section{Methodology - Main Features}

The flowchart in Figure 2 gives an overview of the intended approach. Firstly parts of some generally applied methodology are mentioned, before further specifications are discussed.

As the present lifetime calculations are performed in terms of stresses (Stress-Life Approach) Finite Element Analysis is necessary for the transition of measuring data. In the course of using shell elements for the analysis of welded components two different methods of fatigue life prediction - the nominal stress approach \& structural or geometric (hot spot) stress approach are used and compared.

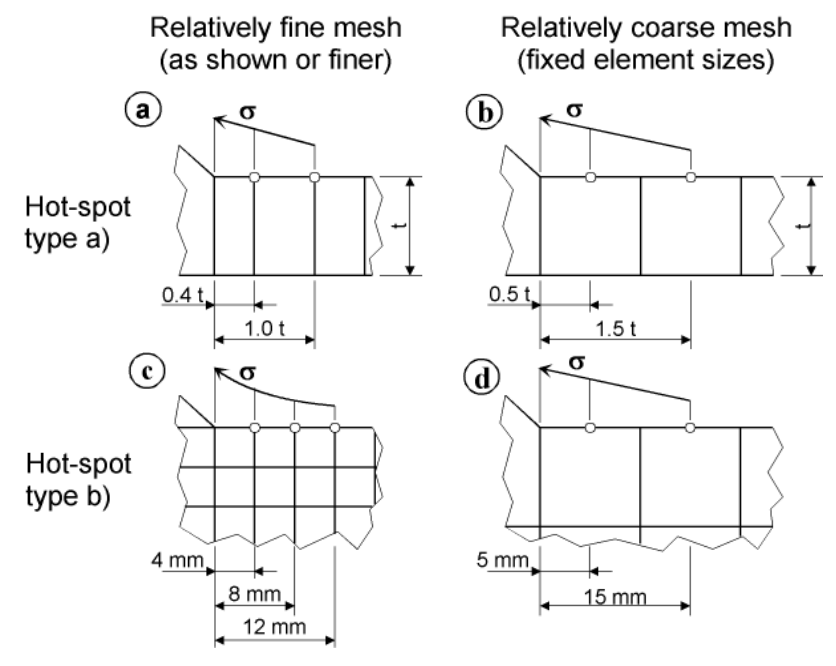

Fig. 2. Meshing and Stress Evaluation using Surface Stress Extrapolation [3]

The elapsed time of the structure's response due to randomly induced traffic loading is recorded by high precision sensor data. An indispensable requirement is to reduce the enormous amount of information of the permanent measuring system to a few statistical data for further assessment. The Rainflow-counting method reduces the sensor data's complete load-time history represented by random sequences of peaks and valleys to a set of fatigue-relevant recurring response-cycles in different categories of intensity and occurrence and has become state-of-the-art in fatigue analysis related with non-periodic loading. As a result we obtain a counting matrix, which shows the amount of occurrences of closed cycles ni from one certain level of displacement to another. By means of Rainflow-counting traffic loading gets anonymous - its results are not directly comparable to conventional traffic counting anymore. The algorithm's principles and specifications used by the authors have already been discussed in [1] \& [5]. 


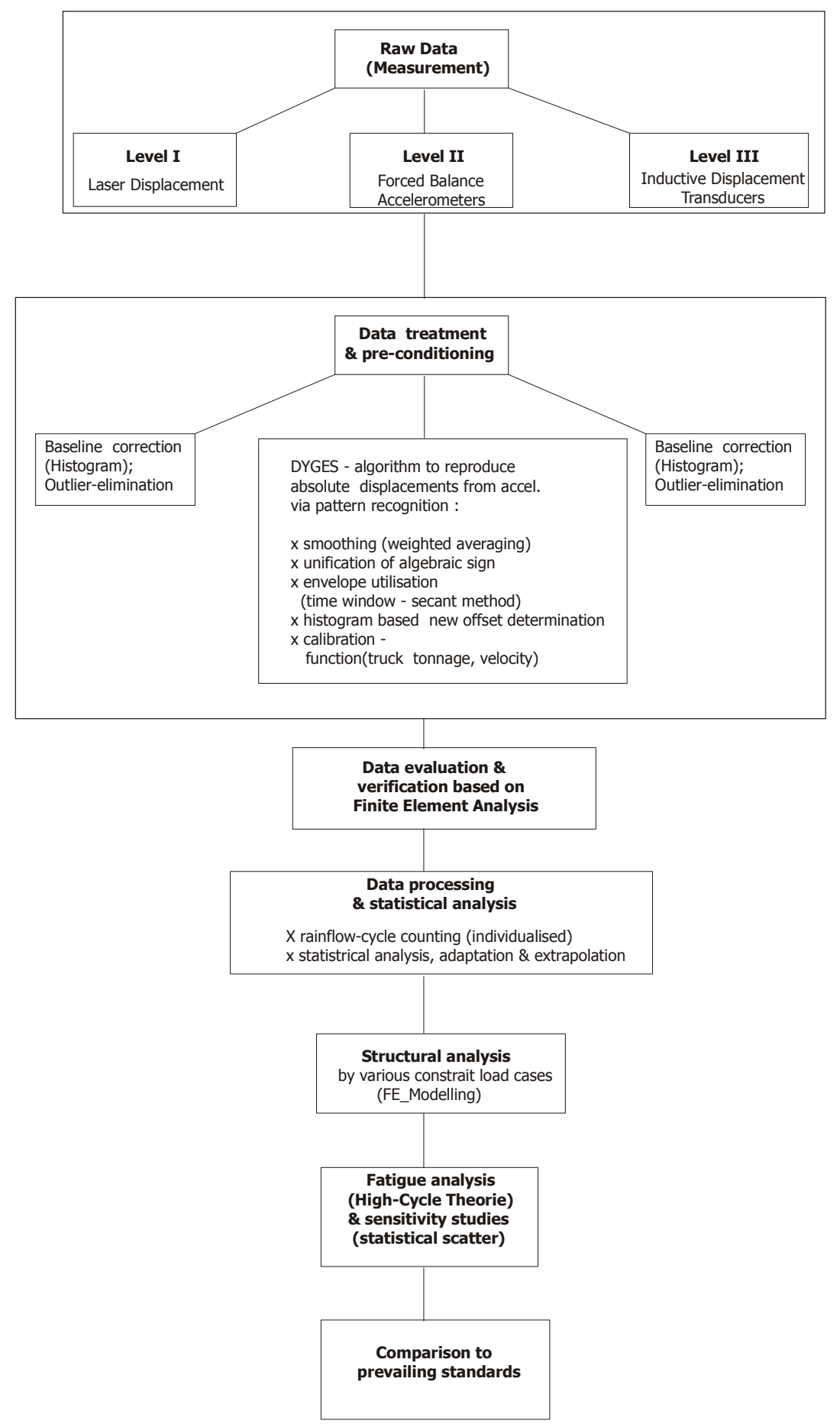

Fig. 3. Detailed flowchart of the methodology

Fatigue analysis by comparing the number of loading cycles ni on a certain stress range-level $\Delta \boldsymbol{\sigma}$ to an allowable number of cycles $\mathrm{N}$ has become a matter of course in civil engineering.

Cases of non-periodic loading as in the present situation demand the well known Modified Damage-Accumulation-Concept by Palgrem-Miner [2].

$$
D_{i}=\frac{n_{i}}{N_{i}} \quad D_{1}=\sum_{1}^{j} \frac{n_{i}}{N_{i}} \quad D_{2}=\sum_{j+1}^{s} \frac{n_{i}}{N_{i}} \quad D=\sum_{1}^{s} D_{i}=D_{1}+D_{2}
$$


Failure is predicted if $D \geq 1$ (inception of a visible crack). The introduced stress life approach is used for long life applications. The fatigue threat exclusively caused by truck traffic leads to the application of High Cycle Fatigue-Theory. Stresses and strains are assumed to remain elastic. This facilitates the calculations, as a single static load case (restraint by a unit displacement) is to be calculated, and related to all other occurring displacements afterwards.

EC 3 - Annex A2 includes suitable provisions, which of its $S-N$ curves ( Figure 4, left) can also be used in connection with the geometric stress approach as an alternative to the curves published in [3].

These standardised stress-life curves are based on graphs derived from experimentally obtained mean-values $\boldsymbol{m}_{\boldsymbol{x}}$, which means, that these data are scattered. Fatigue - as a serviceability-limit state analysis - is therefore defined as exceedance of $5 \%$ probability of failure $\boldsymbol{x}_{5}$, calculated with normal distribution. As this research work tries to encourage in-situ measurements instead of the 'design situations', it is aspired to analyse the consequence of statistical scatter on fatigue resistance.
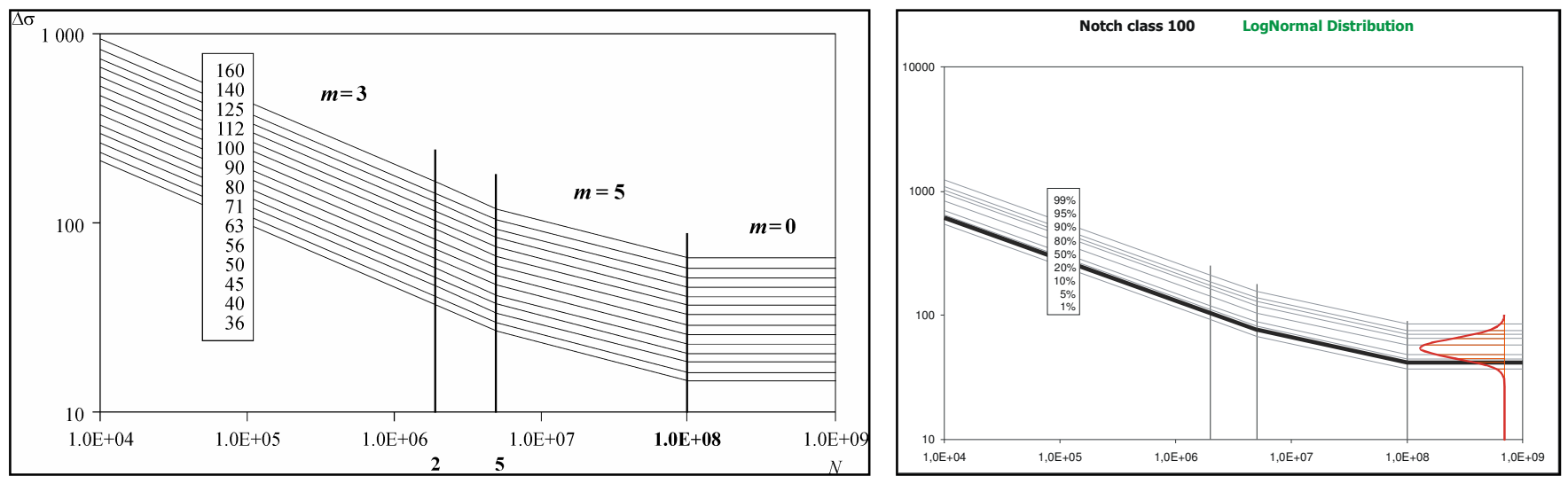

Fig. 4. Statistical scatter of S-N curves

Many methods of statistical evaluation of fatique resistance are available. The authors have chosen an approach according to Spaethe [6], who recommends presupposing a log-normal distribution of stresses at a constant fatigue life, with a certain variance $\boldsymbol{V}_{\boldsymbol{x}}$ increasing with lower notch classes. As fixed variances from standard values are used, no confidence level has to be considered [6]. The characteristic values $\boldsymbol{x}_{p}$, which vary statistically at a specified fractile, are basically determined in two steps (eq. (2) \& (3)):

$x_{p}=m_{x} \pm k_{p} \cdot \sigma_{x}=m_{x} \cdot\left(1 \pm k_{p} \cdot V_{x}\right) \quad x_{5}=m_{x}-k_{5} \cdot \sigma_{x}=m_{x} \cdot\left(1-k_{5} \cdot V_{x}\right) \quad=>m_{x}=\frac{x_{5}}{\left(1-k_{p} \cdot V_{x}\right)}$

The usage of a positive sign leads to characteristic values from 50 to $100 \%$, while the negative sign is used to calculate characteristic values $x_{p}$ between 0 and $50 \%$ probability of failure. $\boldsymbol{k}_{p}$ in eq. (2) represents the standardised, so-called fractile factor, while $\sigma_{\mathrm{x}}$ is the standard deviation.

$\sigma_{u}=\sqrt{\ln \left(1+\left(V_{x}\right)^{2}\right)} \quad m_{u}=\ln m_{x}-\frac{\left(\sigma_{u}\right)^{2}}{2}=\ln \left(\frac{m_{x}}{\sqrt{\left(1+\left(V_{x}\right)^{2}\right)}}\right) \Rightarrow x_{p}=\exp \left(m_{u} \pm k_{p} \cdot \sigma_{u}\right)$ 
After the reference value $\boldsymbol{m}_{x}$ has been derived, eq. (3) describes the transition from normal-distributed to log-normal distributed characteristic values $x_{p}$ for an appropriate description of scattered Wöhler-Curves (Figure 4, right).

\section{Lifetime Prediction on Three Levels}

In long span bridges the load on the primary superstructure is dominated by the dead load. As the fluctuating live load part usually is relatively small, fatigue is of secondary importance. The deck, stringers and transversal girders are mainly subjected to live load and therefore they may be controlled by fatigue. For the sake of completeness the discussion will be focused on three levels, where associated hot spots are determined to determine different levels of relevance (isolation of remaining, fatigue relevant loading cycles $\boldsymbol{n}_{i}$ from randomly occurring traffic).

\section{Level I - Global Behaviour - Measurement \& Applied Procedures}

The following section shows the measurement of vertical displacement of the bridge's main span. It was aspired to obtain measurement data for the global level of analysis. Figure 5 and Figure 6 show schematically, how this was realized. The measuring system consists of a laser-transmitter unit - stationary located at the bridge's abutment - and the receiver unit at a distance of $369 \mathrm{~m}$.

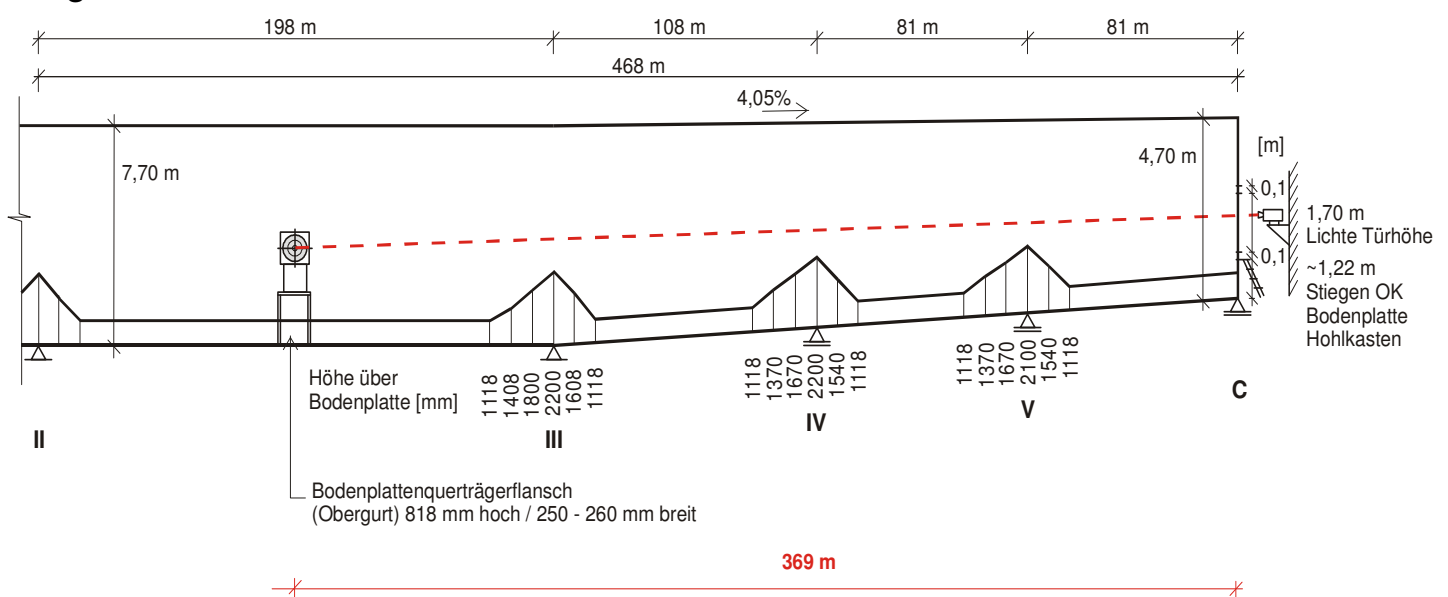

Fig. 5. Schematic representation of the measurement of the main span's vertical displacement

The laser transmitter sends out a narrow, point-type laser beam into a fixed direction. The opt electric receiver measures the position of the optical centre of gravity of the laser beam's hit point on the optical screen. Via framework the receiver is tied nonpositively to the orthotropic bottom plate. Based on the cross-section's contour accuracy, the span's global vertical displacement is measured in a sufficiently precise manner. 


\section{SAMCO}
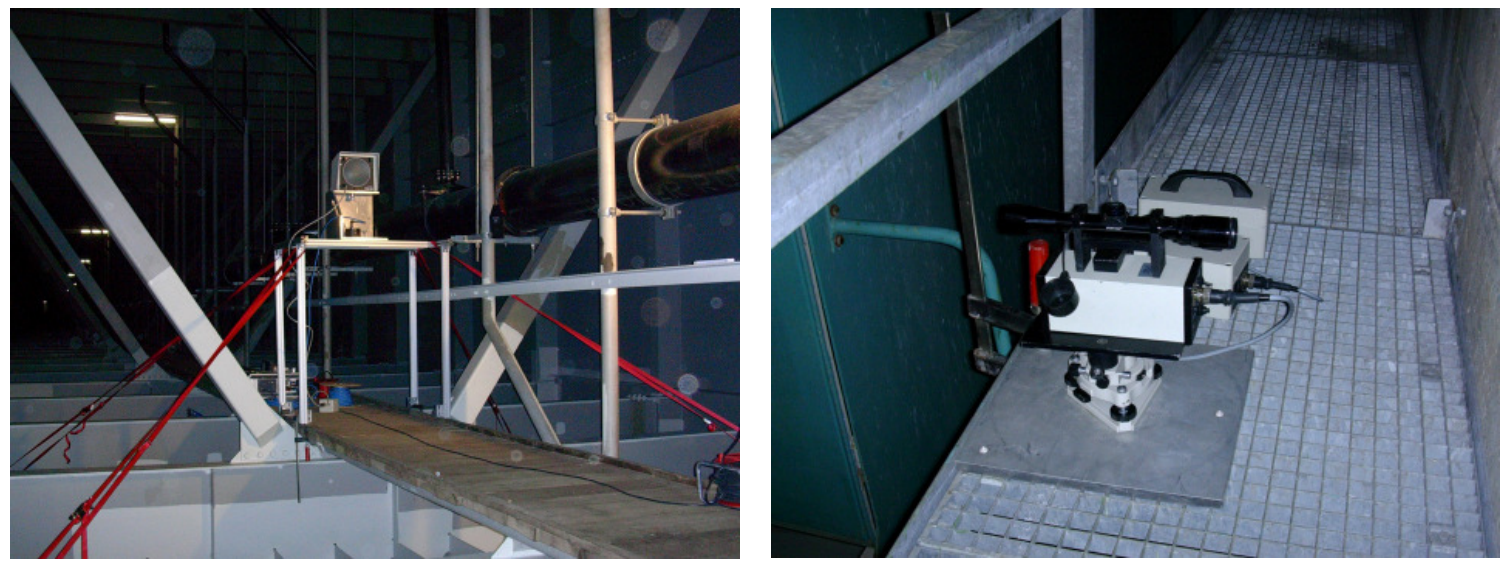

Fig. 6. Optoelectric receiver unit (left) and laser-transmitter unit (right)

In the following the result of a $24 \mathrm{~h}$ measurement campaign is visualised. The total (static \& dynamic) displacement of the span was recognised to be in the range of -12 and $56 \mathrm{~mm}$. The verification of plausibility can easily be performed by analyzing the signal during the night hours (22:00 - 06:00), where regular freight traffic is not allowed to cross the bridge structure. The occurring exceptional truck passages during this time window show vertical displacements in the range of -10 and $18 \mathrm{~mm}$. Static loading by a truck with 35 tons would result in an approximate displacement of $14 \mathrm{~mm}$ (based on a global structural analysis).
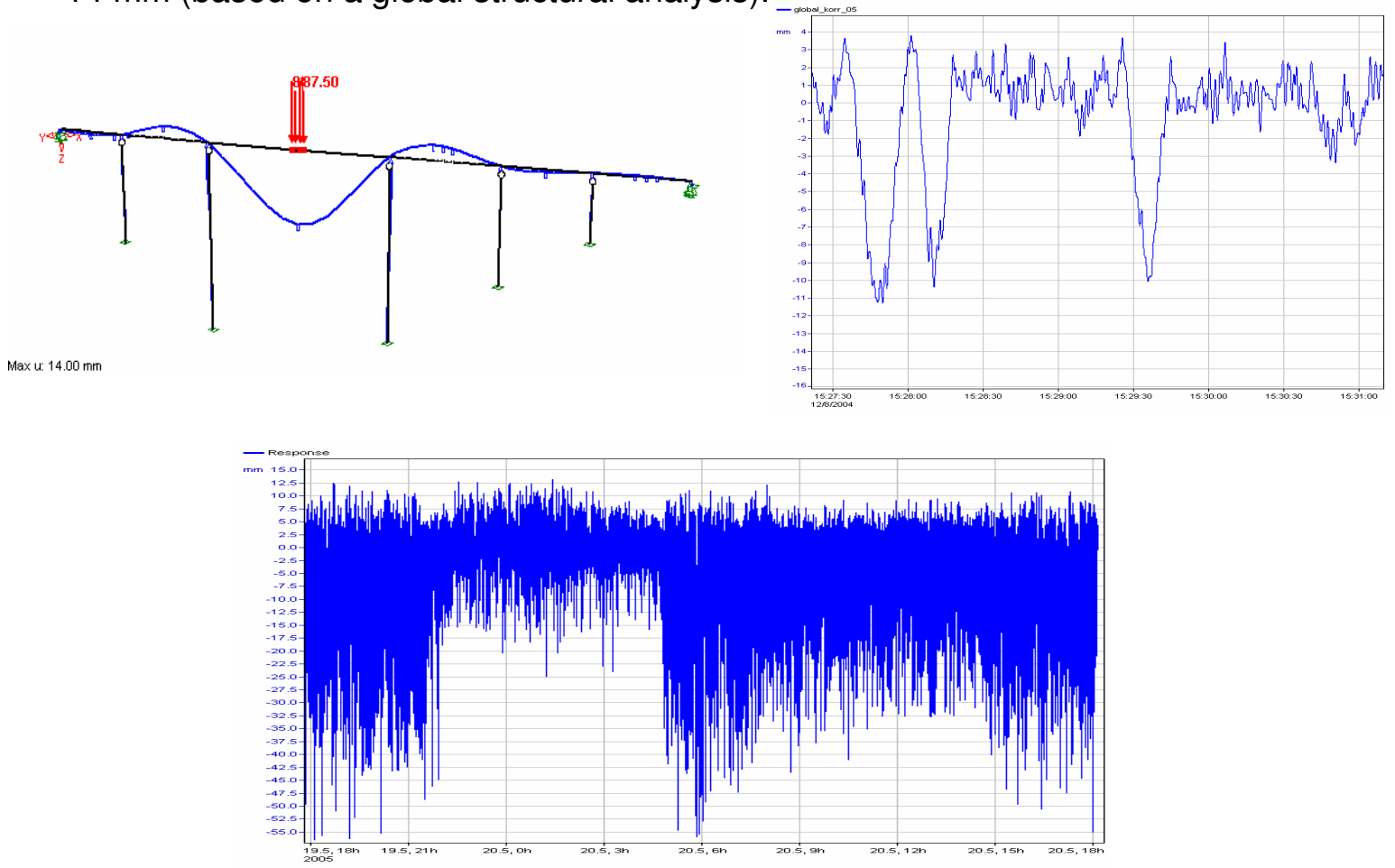

Fig. 7. The main span's vertical response due to traffic loading 19.05.2005 17:50 20.05.2005 18:05

Additionally to quantitative plausibility the qualitative plausibility was also checked. For this reason, some isolated truck passages could be directly verified in the signal belonging to the night hours again. Such passages lasted for $20 \mathrm{sec}$, which corresponds really well with the fact that a truck - maintaining the prevailing speed 
limit of $40 \mathrm{~km} / \mathrm{h}$ - needs approximately 20 seconds to pass the $198 \mathrm{~m}$ long main span.

With regard to serious global stress analysis it was demonstrated, that the desired measurement data were obtained under representative conditions. It needs to be emphasized, that a series of measurements is to be created on several working days by repeating such a measurement campaign to cover the randomness of this individual process.

Figure 8 shows the remaining response cycles of the bridge's main span due to the occurring traffic related with Figure 7 . The fatigue analysis itself is going to be performed in terms of stresses. Firstly the chosen approach demands some global structural analysis. The obtained displacement cycles are implemented into a global framework model of the whole bridge by means of a single constraint load case (Figure 9, left) with RSTAB. System identification performed with this software compared to measurements showed, that the global behaviour is represented quite well. On the other hand fundamental phenomena like the effective width, which is of eminent importance for accurate stress analysis, are disregarded with RSTAB. To overcome this problem, the following mechanical solution was developed:

As already shown, the measured displacement cycles are implemented into a global framework model of the whole bridge by means of a single constraint load case. Subsequently a $63 \mathrm{~m}$ long bridge segment - modelled with shell elements (RFEM) is prepared (Figure 9, right), which can be individualised (in its geometry) depending on the determined area of interest. The transition from the framework based state variables to the FE (shell element) based ones is realized through assignment of displacements and cross sectional rotations from the global bridge model along the outer edges of the modelled bridge segment (Figure 9, right). The applied hypothesis for the implementation of measured, global displacement data on the global bridge model via unit load case (restraint at the sensor position) has to be confirmed. For that reason further fatigue calculations are to be extended to other possible loading configurations - with the same displacement value at the sensor position, but with other flexion shapes - leading to local analysis corresponding with different notch classes. In the same way, loading configurations related with explicit torsional impact - and in accordance with the measured vertical displacement - must be determined. This need is motivated by some approximate investigation as well as inspections, which have already revealed, that the box girder's torsional bracings (square bar diagonals) represent a potential weakness, thus they are going to be one of the main global areas of interest.
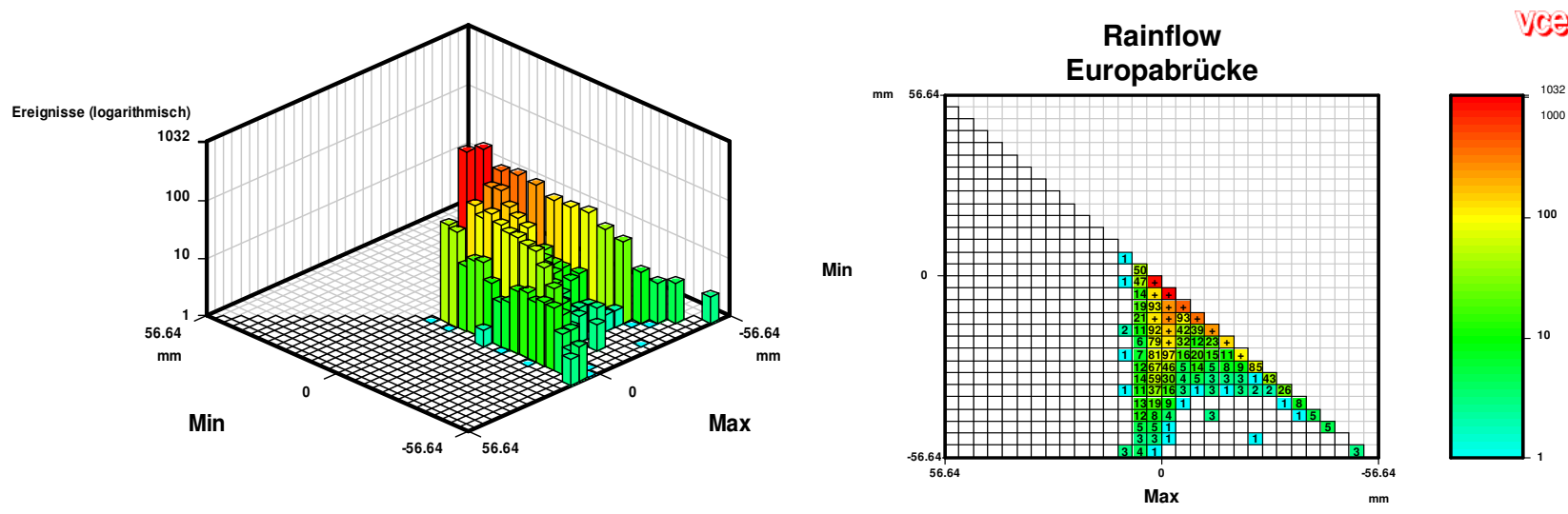

Fig. 8. Rainflow-Counting of global bridge displacement 


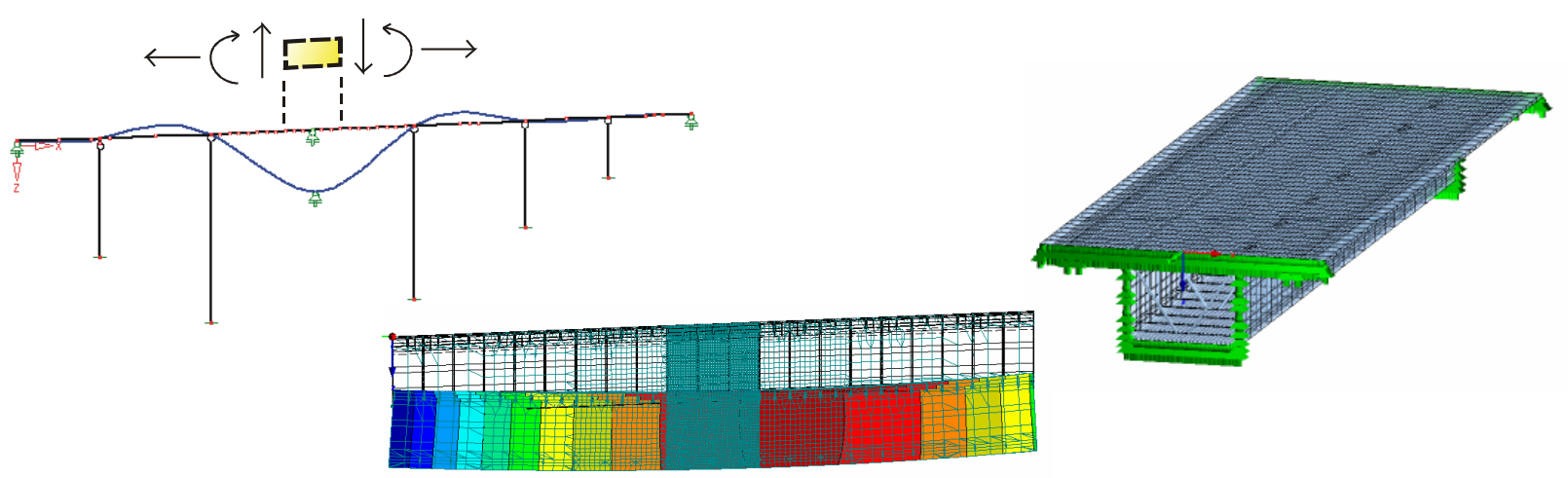

Fig. 9. Structural analysis model under constraint of the unit load case

During numerous inspections and measurements, it has also been recognised that the impact of trucks - suddenly entering the bridge's first span in the downhill direction - cause noticeable global vibration. Therefore global fatigue investigations will have to be made for the first span, following the procedural principles described for the main span.

A transition from the Rainflow Matrix to a so-called Damage Matrix is necessary to follow up the consequence for fatigue. Depending on the measured time frame the created Rainflow Matrix is extrapolated to a period of one year. After having assigned the area of interest to a certain notch class, the significance of all elements of the matrix in terms of stresses can be determined by its partial damages $\boldsymbol{D}_{\boldsymbol{i}}$, accumulating to a total damage $\boldsymbol{D}$ per year. Thus, for the Damage Matrice's vertical axis terms of frequency of occurrence are replaced by damage rates $r$ (eq. (1)).

To get a better understanding of every element's damage relevance $r$, eq (4) is used to transform loading cycle counting matrices into damage assessment or damage quantification matrices [1].

$$
r=\frac{D_{i}}{D} \cdot 100 \quad[\%]
$$

\section{Observance of the Randomness of Several Individual Measurements}

Several planned global measurement sessions repeated under equal conditions represent a random sample from the universal set for global behaviour. For reasons of complexity and economy this laser-supported measurement configuration is not capable of being a part of the permanent monitoring system. To cover the randomness of these several individual measurements, an approach from Bronstein et al. [7] is chosen. Ultimately the confidence interval for the measured mean value with an unknown variance is derived. Following the hypothesis in [7], the random variables obtained from such individual measurements follow the GaussianDistribution regardless of the universal set's distribution function. Thus confidence intervals for the obtained measurement data are established by means of an accepted, predetermined probability of error $\alpha / 2$ (eq. (5)).

$$
\mu=\bar{x} \pm \frac{s}{\sqrt{n}} \cdot t_{\omega 2 ; n-1}
$$


$\mu$ represents the modified reference value (e.g. accumulated damage $\mathrm{D}$ - but could also be the Rainflow procedure's section values leading to damage $\mathrm{D}$ ) belonging to individual measurements. $\bar{x}$ is the measurement-based mean value, $s$ is the measurement-based standard deviation, $\boldsymbol{n}$ is the amount of performed measurements and $\mathbf{t}_{\alpha / 2 ; n-1}$ is the quantile corresponding to a $t$ - distribution.

\section{Level II - Cross Sectional Behaviour}

The bridge's 7,5 m long cantilevers are primarily heavily loaded by trucks, as those with a permissible maximum weight of more than $7,5 \mathrm{t}$ are only allowed to use the first lane. This emphasizes the importance of this level; therefore it was decided to incorporate the cantilever itself into the permanent monitoring system.

\section{Measurement \& Applied Procedures}

For permanent monitoring of the cantilever vibration, so-called Forced Balance Accelerometers (FBA11, sampling rate $100 \mathrm{~Hz}$ ) are used. Those transducers register the alteration of electrical capacity (by means of voltage) based on the variation of their position due to a springy mass - influenced by acting accelerations. According to Fig. 10, three one-dimensional sensors were installed in the region of bridge pier II to describe the loading behaviour in the transverse direction. Two of them are located in a defined distance of $15 \mathrm{~m}$ - in the same direction of traffic - to verify recurring truck passages and their related velocity without any disturbance of traffic by smoothing the signal with various time intervals (weighted averaging) and subsequent automatic "peak-picking". This procedure is comparable with the utilisation of digital filters and widely eliminates the influence of interferences in the higher frequency range. Nevertheless it was found out that smoothing maintains the whole energy impact much better than digital filters, especially in case of trucks passing the measurement section back to back.

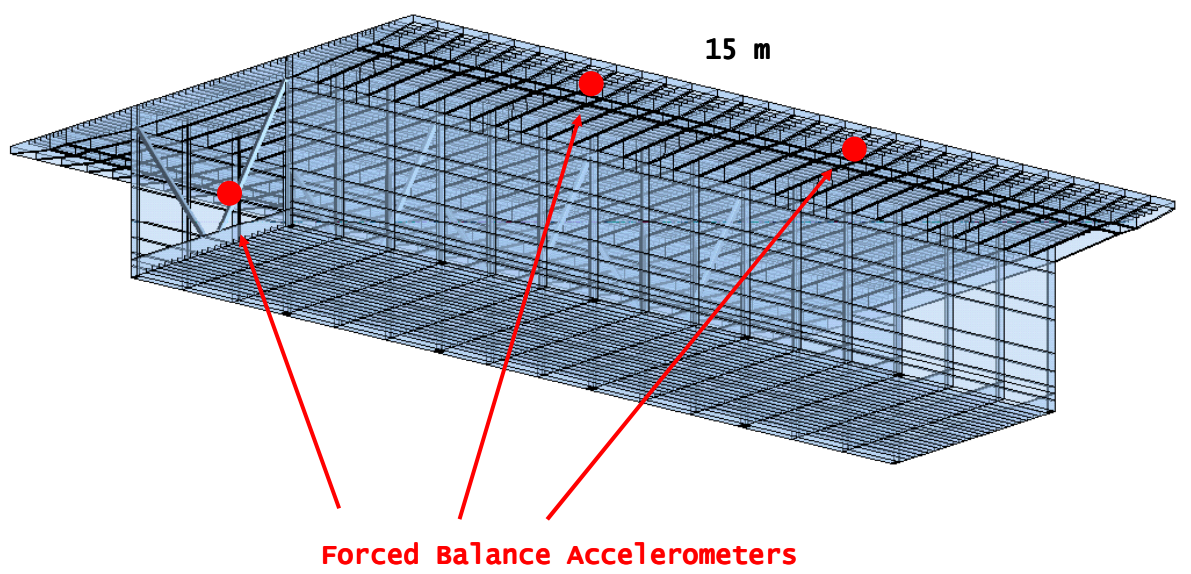

Fig. 10. Vehicle recognition by acceleration sensors placed successively

Consequently the structural member's response - the sensor signal of acceleration has to be transformed into a signal of absolute displacement, as this is the decisive variable of interest for further stress-based fatigue analysis. This state variable typically can not be derived mathematically from transducer-accelerations. Elimination of the acceleration's offset and double integration lead to vibration about the neutral axis - in terms of relative displacements [5].

For that reason the so called DYGES algorithm (the German acronym for Dynamic Weight Registration System) was developed, which enables the authors for the first 


\section{SAMCO}

time to generate absolute displacements from relative accelerations by means of digital signal processing.
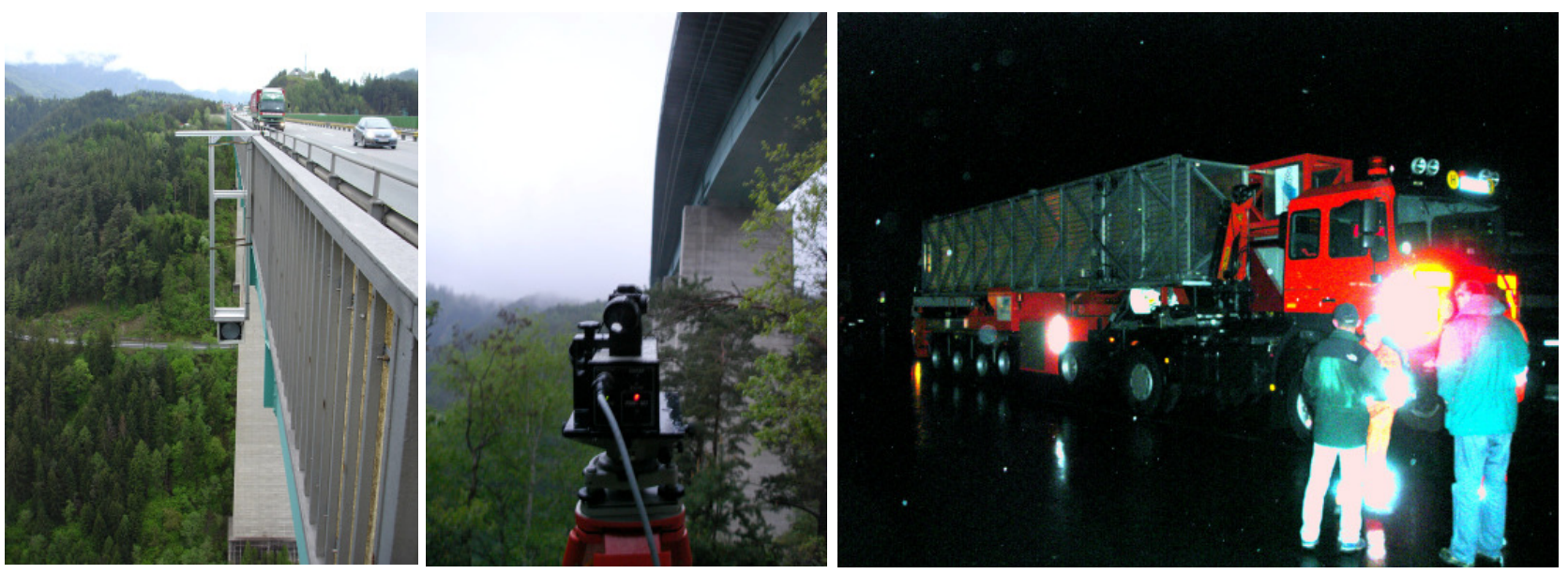

Fig. 11. Laser transmitter unit; optoelectric receiver unit, fully loaded calibration truck $(42,8 \mathrm{t})$

This pattern recognition based procedure mainly consists of weighted averaging, unification of the signal's algebraic sign (squaring), an envelope function (based on time window secant method) and a histogram-based new offset determination (Figure 3).

Results from the current state of the transformation algorithm are typically visualised in Figure 19 - the influence of prevailing velocities and motor vehicle tonnage is already considered. It is to be emphasized, that this approach of deriving cantileverdisplacements due to traffic loading is a structure-specific approximation, which had necessarily to be optimised by calibration truck-crossings for consistent analysis. This information was obtained in the course of an additional measurement campaign in May 2005, which was conducted under the following aspects: Truck with varying, well-known loads (a fully loaden truck and also a truck without any tonnage) passed the measurement section with stepwise increased velocities (from 20 to $60 \mathrm{~km} / \mathrm{h}$ ). This process was repeated under simultaneous laser-displacement and accelerometer measurement. The essential sources of interest had necessarily to be implemented into the DYGES procedure, were the dynamic amplification due to the influence of transported weight as well as due to the influence of the truck's velocity.
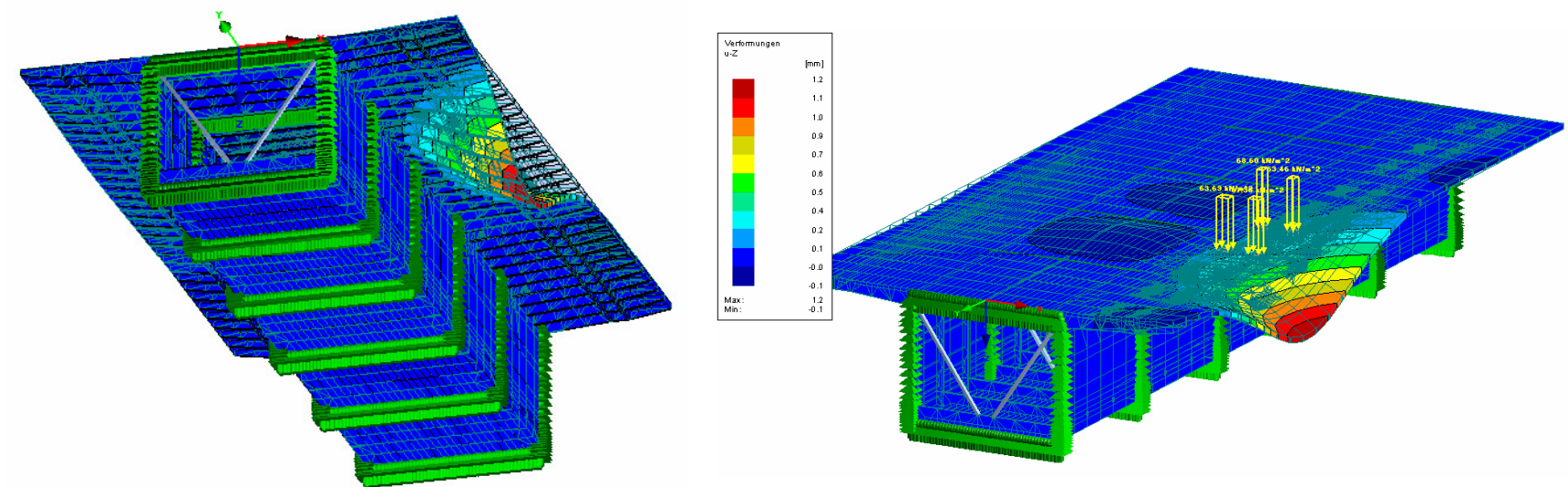

Fig. 12. Bridge segment modelled with shell elements and stressed by an unloaded truck $(8,7$ tons) 
The measurement section is located directly above bridge pier II, thus the analytical calculation (for verification purposes) with the same (static) truck loading was carried out on a bridge segment modelled with shell elements under prevention of global deformations (Figure 12 \& Figure 13). The achieved cantilever deformations for the unloaded as well as for the loaded truck show a very good accordance with the obtained measured values (Figure 14). When analyzing Figure 12 and Figure 13, one decisive assumption of the DYGES - algorithm is confirmed analytically. The cantilever's response stressed by explicit truck loading does not interact with the cantilever belonging to the opposite driving direction. This fact supports the already made observance during extensive measurement data analysis.

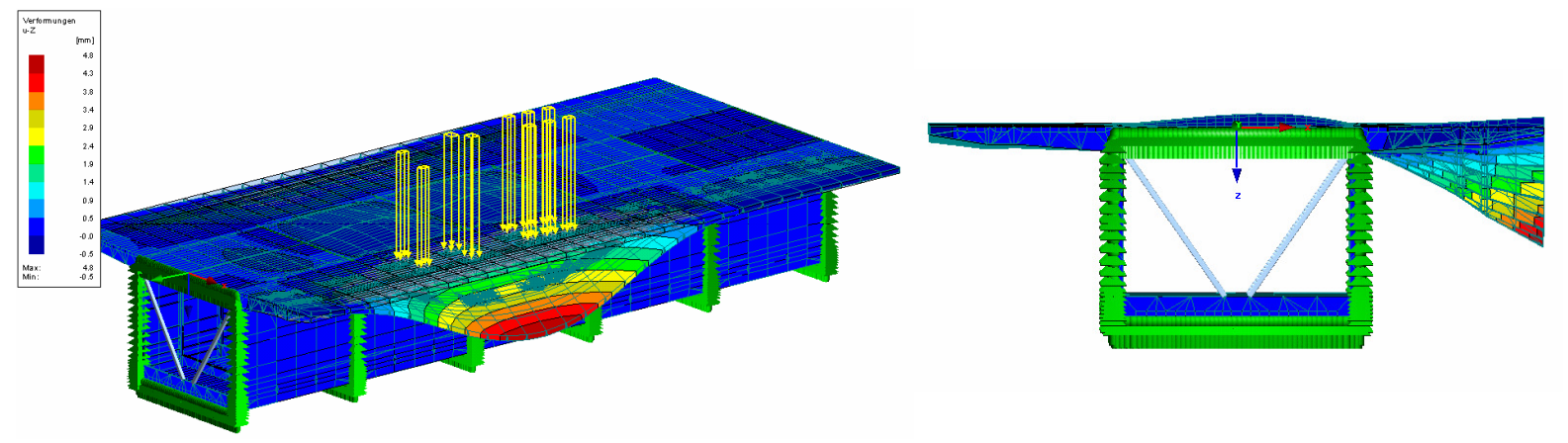

Fig. 13. Bridge segment modelled with shell elements and stressed by a heavily loaded truck (42,8 tons)

Figure 14 shows a very remarkable result of the laser supported calibrationmeasurement campaign. The pattern was obtained by weighting both performed truck-crossing repetition procedures. The functions, which can be understood as an amplification- factor pattern, have similar characteristics for heavy loaded trucks as well as for unloaded ones, even if the latter one is more distinctive.

Cantilever calibration truck-crossings Europabrücke

Cantilever calibration truck-crossings Europabrücke
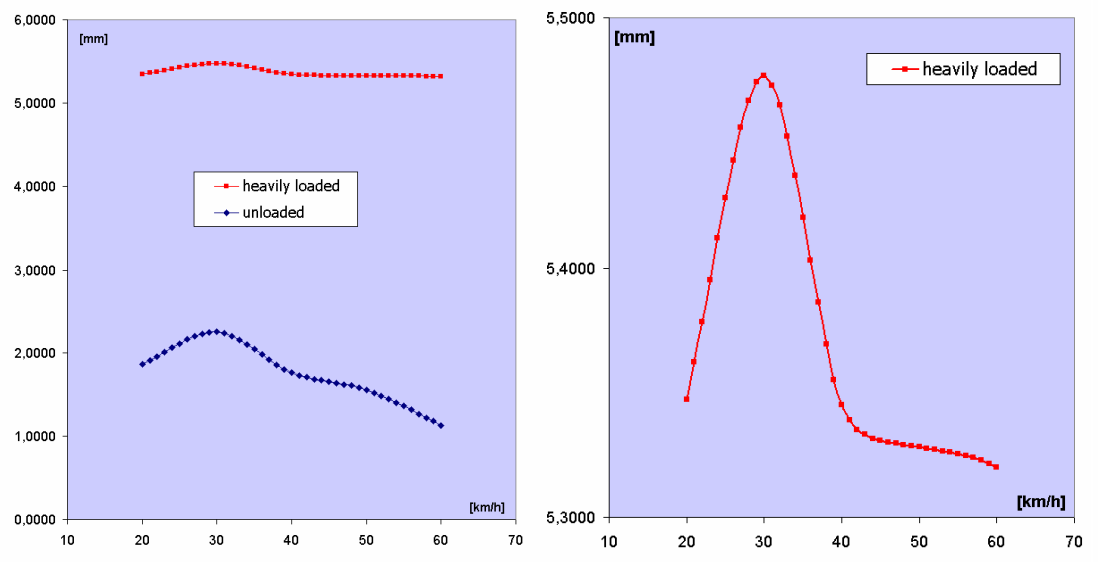

Cantilever calibration truck-crossings Europabrïcke

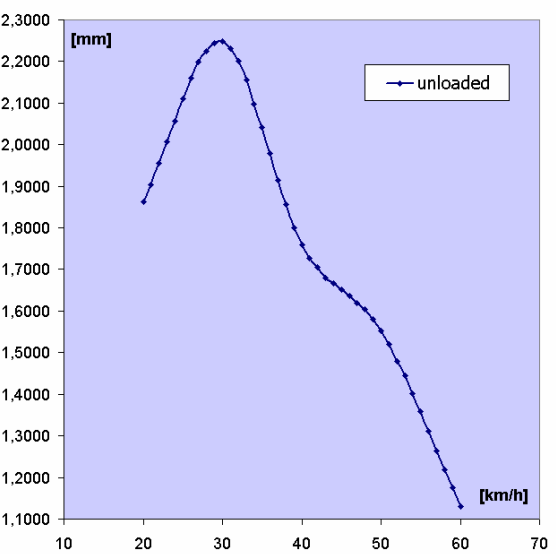

Fig. 14. Amplification pattern (laser supported absolute displacements) of the cantilever

Long term monitoring based fatigue investigation (High Cycle Fatigue) assumes stresses and strains to remain elastic. For that reason these two functions can be connected via generatrix (linear interpolation), which enables to utilize the information for an automatic registration of all truck weights occurring under characteristic operational conditions. 


\section{$S_{A M C O}$}

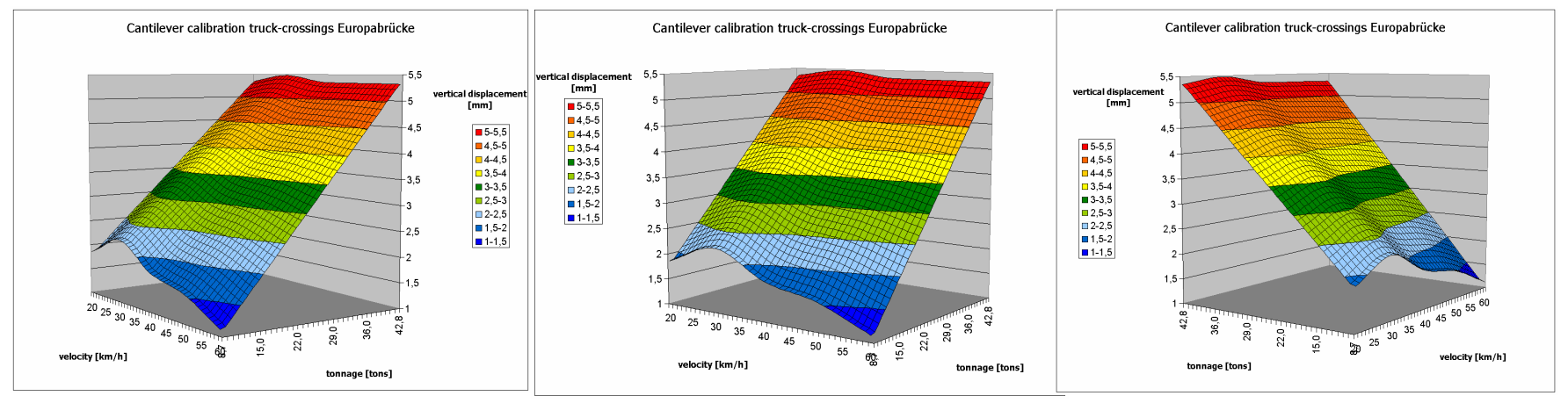

Fig. 15. Areal function for absolute vertical cantilever displacements (laser) with varying weight and velocity

Figure 16 gives the complementary information to the results visualised in Figure 14 for simultaneously performed, accelerometer-supported cantilever measurements. The patterns were again obtained by weighting the DYGES based results of both performed truck-crossing repetition procedures (Figure 3 ). The functions, which can be understood as an amplification- factor pattern, again have a distinctive trend line for heavily loaded trucks as well as for unloaded ones, even if each flexion shape is an individual one in detail.
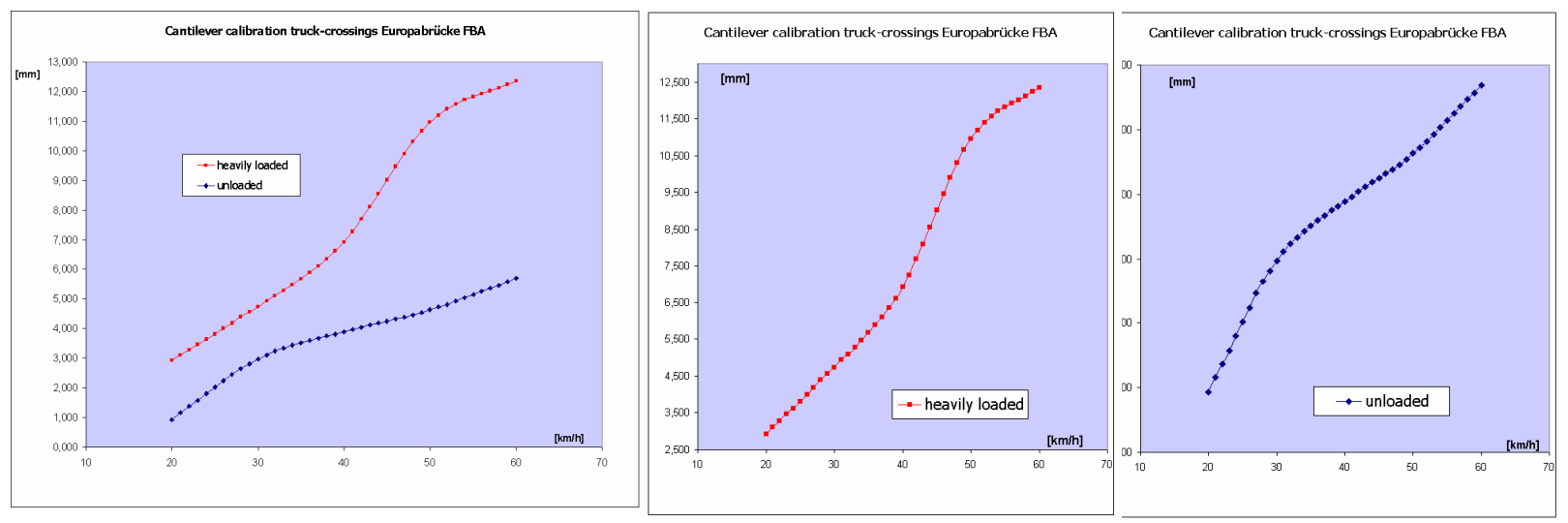

Fig. 16. Amplification pattern (accelerometer supported non-calibrated absolute displacements) of the cantilever
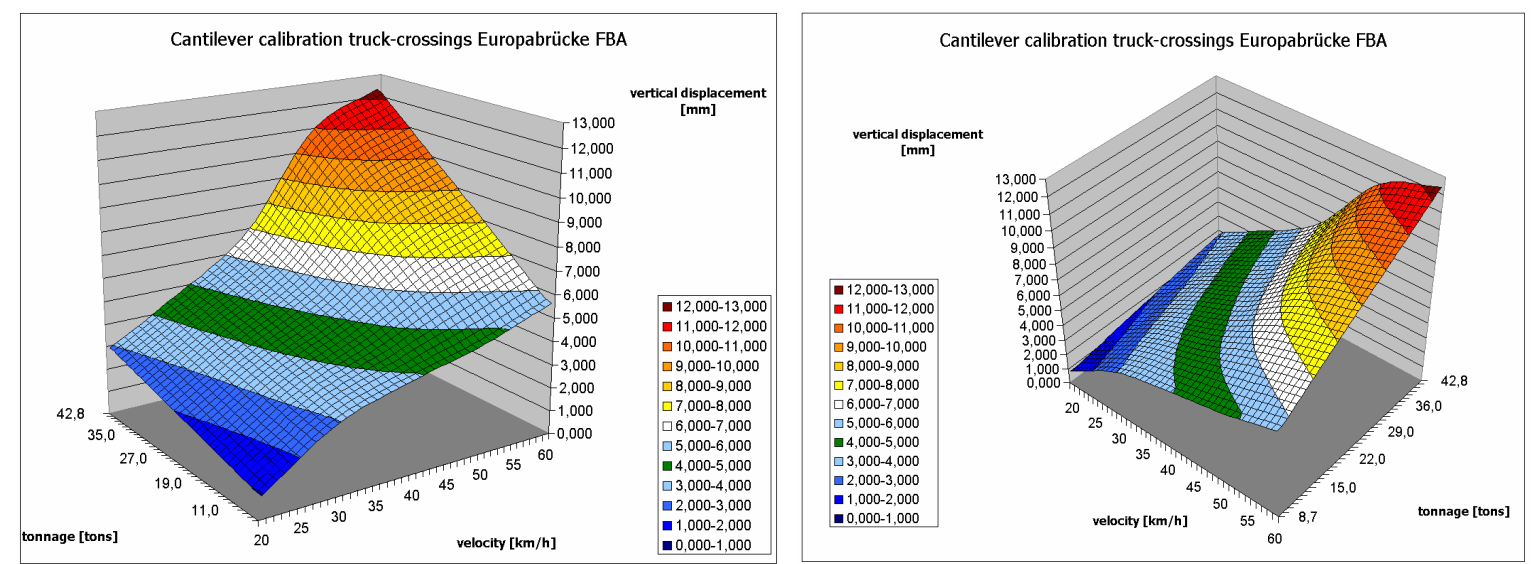

Fig. 17. Areal function for reproduced absolute vertical cantilever displacements (accelerometer) with varying weight and velocity 
Successively these two functions are connected via generatrix (linear interpolation), which enables to utilize the information for an automatic registration of all truck weights occurring under characteristic operational conditions (Figure 17). Especially the comparison of both derived areal functions (Figure 15 \& Figure 17) strongly supports the need to implement a calibration sequence into the developed DYGES algorithm. Thus a scaling matrix is derived from both matrices, which fulfils this requirement to calibrate the reproduced, accelerometer-based absolute displacements.

Of course the output of the calibration measurement campaign is limited in terms of the registered truck's weight and velocity. Regarding to the truck weight it can be assumed, that values lower than 8,7 tons (related with approximately $1,5 \mathrm{~mm}$ cantilever deformation) do not exceed the Wöhler curve's cut-off limits. Values higher than 42,7 tons should not be exceeded remarkably, as extended observation by the bridge owner confirmed compliance with the permissible maximum weight (44 tons).

Regarding to the truck velocity it can be assumed, that values lower than $20 \mathrm{~km} / \mathrm{h}$ are negligible while values higher than $60 \mathrm{~km} / \mathrm{h}$ are not possible during the daytime. This assumption is based on an existing speed limit $(40 \mathrm{~km} / \mathrm{h})$ but is also a logical conclusion of the fact, that the prevailing intensity of traffic flow prevents values higher than $60 \mathrm{~km} / \mathrm{h}$.

Long term monitoring - leading to an automatic registration of truck volume, truck velocity and truck weight - will state more precisely, how often exceedance of 60 $\mathrm{km} / \mathrm{h}$ will occur during the night time. In any case Figure 17 and Figure 18 clearly show, that the usage of upper bound values of the scaling matrix at least lead to conservative solutions - for exceedance cases of velocity - even if the exact information for rescaling is not available.

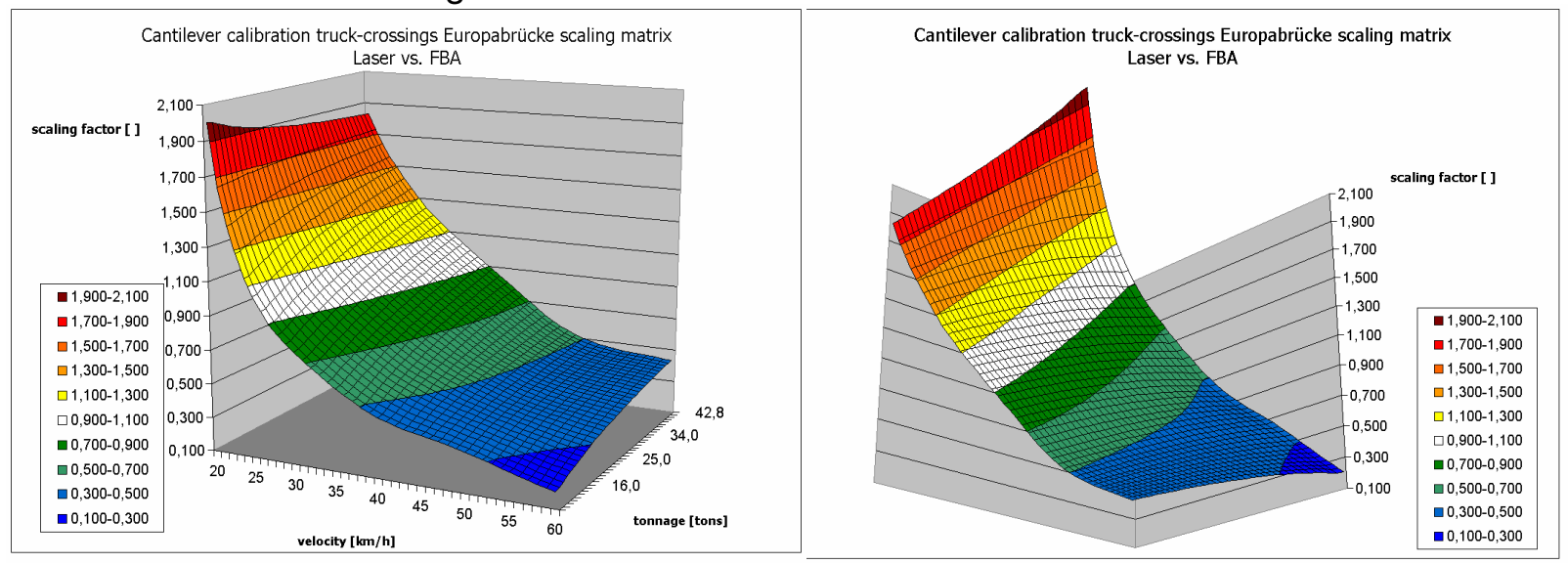

Fig. 18. Scaling matrix to calibrate reproduced absolute vertical cantilever displacements (accelerometer $=>$ laser) in dependence of varying weight and velocity

Following the described principles the cantilever's vertical deformations are reproduced qualitatively (progression) and quantitatively with sufficient accuracy for further fatigue assessment. 

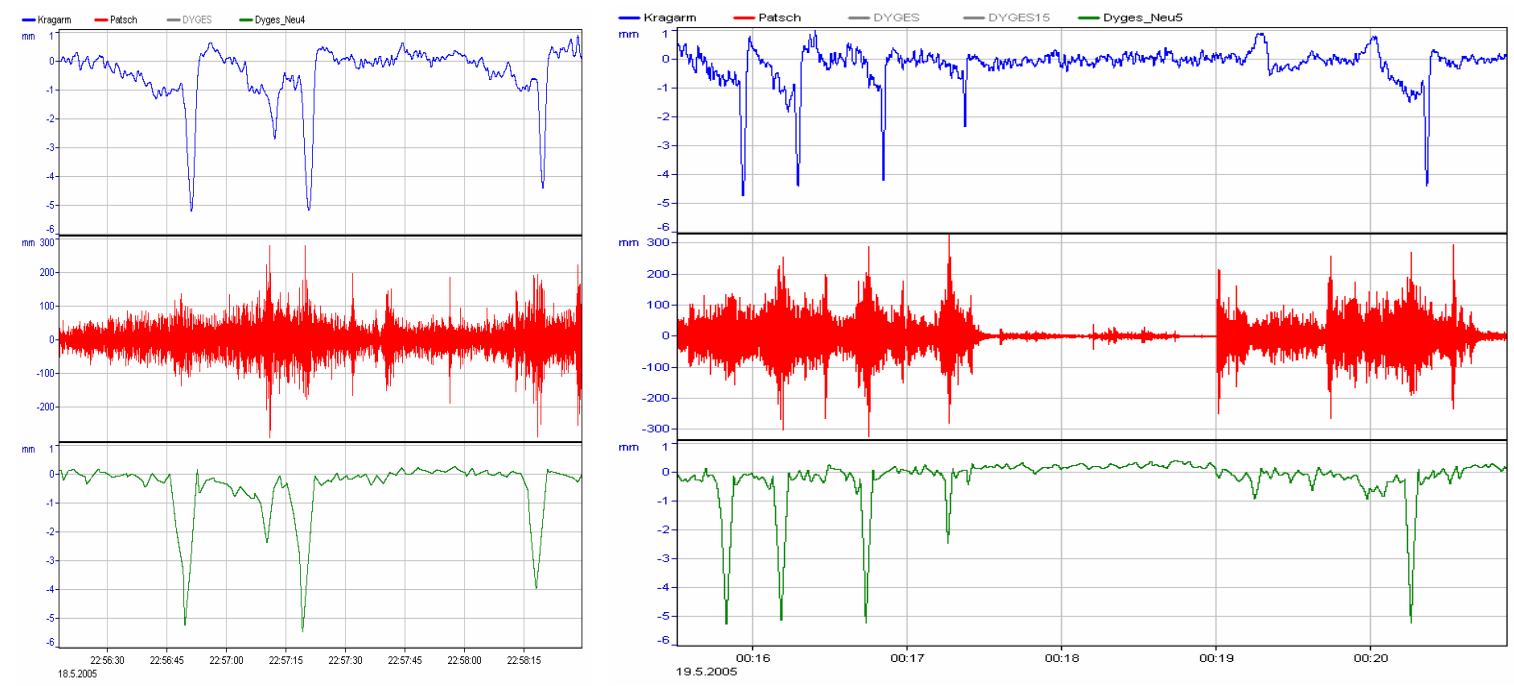

Fig. 19. Reproduced cantilever displacements (green) vs. directly measured ones (blue) \& acceleration signal (red)

A detailed observation of Figure 19 shows two main perceptible deviations between the original (blue) and the reproduced (green) qualitative displacement pattern. With regard to continuative fatigue analysis their influence on the obtained data can be neglected and is explained in the following: Existing cycles - mainly occurring during unloaded vibration about the neutral axis - that do not exceed the predefined, correlation matrice's section margin are ignored.

The imaginary flow of water, which is initiated in every peak and valley in the course of rainflow counting, is followed until it experiences a drop. Referring to the principles of the applied Rainflow algorithm ([1] \& [5]), there is no discrepancy, when small pattern-sequences are not available anymore, as long as all occurring local extreme values are made available for further analysis. Both cases do not represent a source of uncertainty for further fatigue-relevant cycle-counting.

Figure 20 shows the result of Rainflow counting for a monitoring period of one month based on the present stage of development of the introduced reproduction procedure for cantilever displacements (DYGES). The influence of all levels of loading is obvious but eliminated via fatigue relevant transition to a corresponding Damage Matrix.
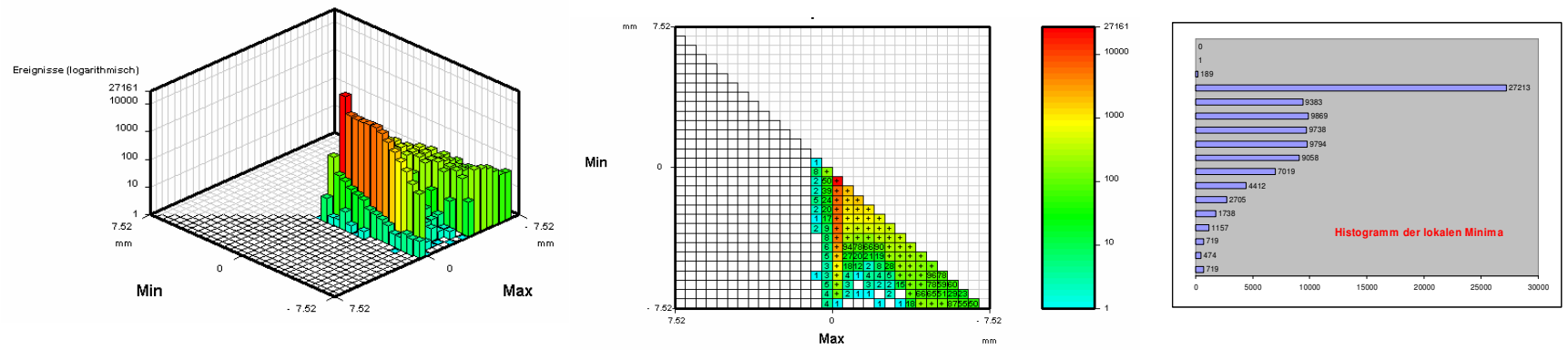

Fig. 20. The axonometric projection of the Rainflow Matrix (left), its relating ground view (right) and the appropriate level crossing histogram for a representative month (July 2004)

\section{Adaptation of Rainflow Matrices}

The derived counting matrices have to be adapted for reasons of necessary extrapolation and superposition. The Europabrücke was subsequently equipped with the described permanent measurement system. Even if the actual performance is measured over a long period of time, this timeframe is relatively small compared to 
the bridge's overall lifetime, resulting in the need for extrapolating the derived data. Studies such as the one by Dreßler et al. [8] have shown that an extrapolation of Rainflow Matrices due to the detailed knowledge about the progression of traffic volume by uniform scaling of the number of occurrences is not adequate for lifetime estimations. The authors have chosen a method developed for the usage in the Rainflow domain - based on [8], which covers the remaining scatter of prevailing loading spectra - assuming that the physical process corresponding to the extrapolated matrix is not of a significantly different type than the process generating the initial matrix.

The basic concept of nonparametric density estimators assumes that a certain set of measured discrete points is described by a function. $f(x)$ depends on data values $\boldsymbol{Y}_{\mathrm{i}}$ measured at points $x_{\mathrm{i}}(\mathrm{i}=1, \ldots \mathrm{N})$ with a random error $\varepsilon$.

$$
Y_{i}=f\left(x_{i}\right)+\varepsilon_{i}
$$

$f(x)$ is estimated by the mean value and weighted by the function's smoothness.

$$
\overline{f(x)}=\frac{1}{N} \sum_{j=1}^{N} w_{j}(x) \cdot Y_{j}\left(x_{i}\right)
$$

In one-dimensional cases, the Gaussian kernel estimator (eq.(8) ( 8)) would be used.

$$
k_{\sigma}(u)=\frac{1}{2 \pi \sigma} \cdot e^{-\frac{u^{2}}{2 \sigma^{2}}}
$$

However, Rainflow histograms have an arbitrary shape, which can only be described by a nonparametric approach. Eq. (9) is introduced for two dimensional purposes to transform the discrete Rainflow Matrix into a smooth function, which is more readily accessible.

$$
k_{\sigma}(u, v)=\frac{1}{2 \pi \sqrt{\operatorname{det} \Sigma}} \cdot e^{-\frac{1}{2}(u, v) E^{-t}\left(\begin{array}{l}
u \\
v
\end{array}\right)}
$$

$\boldsymbol{k}_{\boldsymbol{o}}(\boldsymbol{u}, \boldsymbol{v})$ describes the adaptive, two variate density estimator (normal distribution, mean $=0$; covariance matrix $\Sigma$ ), which reflects the mechanical background of the "from-to" Rainflow Matrices as well as the characteristics of fatigue relevance within a counting matrix.

$$
\Sigma=\frac{1}{2} \sigma^{2}\left(\begin{array}{ll}
\lambda^{2}+1 & \lambda^{2}-1 \\
\lambda^{2}-1 & \lambda^{2}+1
\end{array}\right)
$$

The choice of $\Sigma$ reflects the special need that entries on the same subdiagonal should have a higher influence, than the ones on other subdiagonals. As visible in Figure 21, the contour lines of the kernel then describe ellipses where the principal axis is parallel to the subdiagonals and $\lambda$ provides the ratio between the large and 
the small principal axis [8]. $\sigma^{2}$ is going to be chosen individually for each entry to guarantee that there are enough values to perform a statistically good averaging.

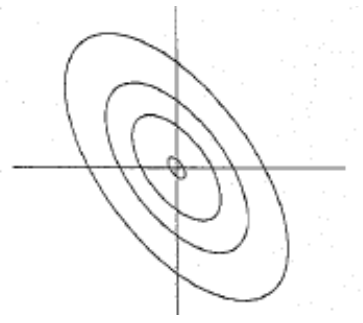

Fig. 21. Some contour lines for $\lambda=2[8]$

Assuming that several derived counting matrices are generated by equal subdivision and assignment of entries, they can be superposed linearly. Such Rainflow Matrices correspond to several loading histories, stringed together. This enables the authors to consider changing service conditions like the variation of the loading level possibly by re-scaling of the Damage Matrice's subdivision.

\section{Fatigue Analysis in Terms of Stresses}

Figure 10 has already shown a certain bridge structure segment, where the cantilever acceleration sensors are installed. Shell elements are going to be used in the course of FE analysis to model the middle plans of the plates. Plate thickness is given as a property of the elements. The structure will be modelled with a coarse mesh - therefore a refinement at the relevant hot spot areas is necessary.

The authors would like to point out the following conclusions as a result of detailed investigation for this level of fatigue-analysis: The appropriate model of the hot spot areas includes corner arcs for welds as well as additional stiffening plates for connector units. The weld geometry itself is neglected. The location of weld toes at structural intersections represents a very conservative solution for the stress extrapolation. The physically imprecisely modelled overlapping area can alternatively lead to the consideration of an offset (half of the corresponding plate thickness), before the relevant values for stress extrapolation are determined.

\section{Level III - Local Systems}

Comprehensive experience with fatigue problems, that has been made in civil engineering, lead to provisions in standards, that this level of analysis could be an endangered one. This research work tries to encourage the target of developing innovative methods in each level of analysis. Therefore the following concept was prepared to asses the fatigue threat on a certain bay of the orthotropic deck (Figure 22). 


\section{Measurement \& Applied Procedures}

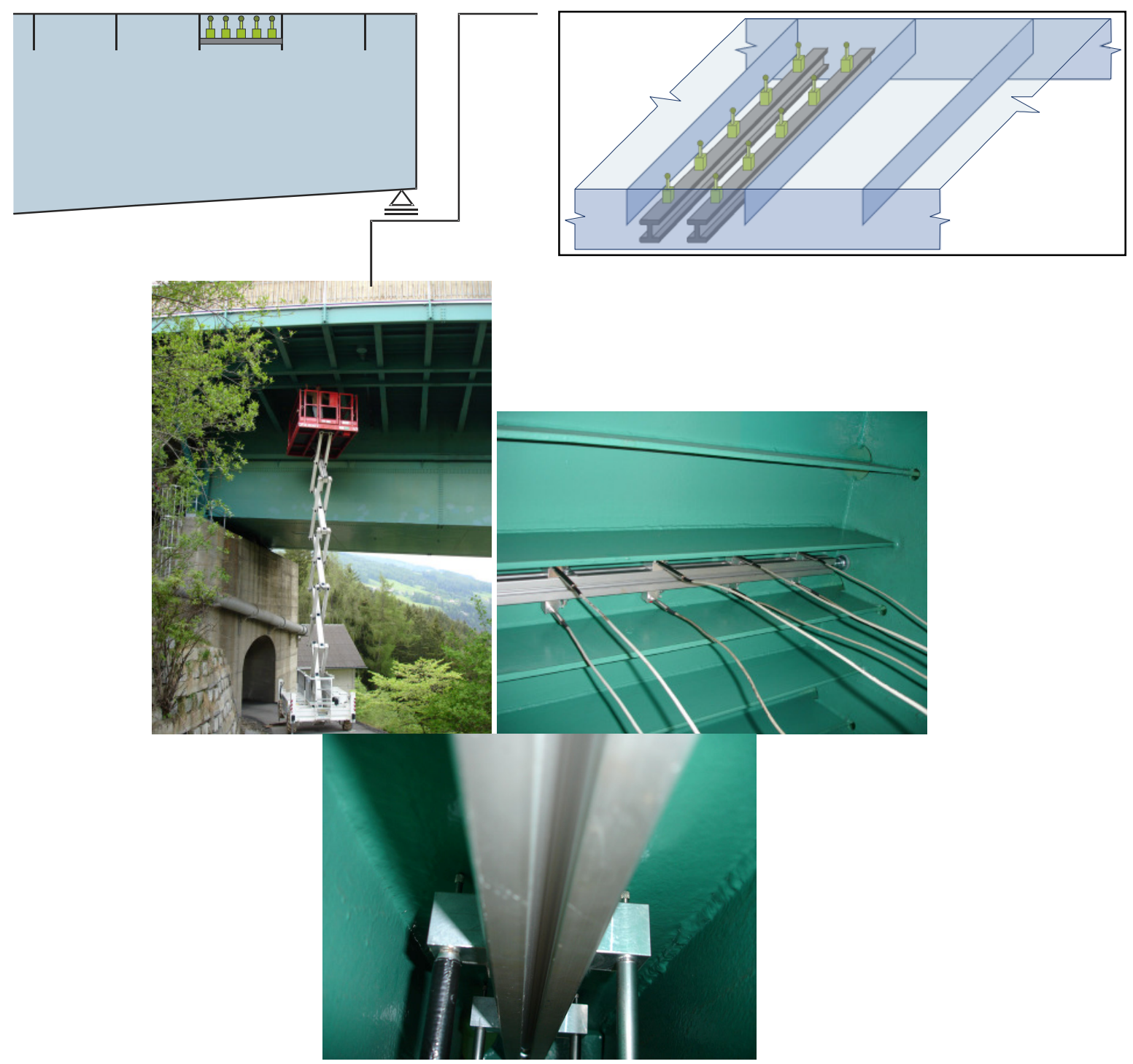

Fig. 22. Survey of a certain bay's deformation (orthotropic deck)

Contrary to numberless assessment of notch details by strain gauges, an areal measurement grid of ten inductive deformation transducers - distributed in two rows registers the response of the bay-plate due to tyre-loading. These transducers follow the LVDT-principle (linear-variable-differential-transformation) and are equipped with a restoring spring to register variation of lengthwise deformation. For the purpose of a better accessibility the measurement grid was located near a bridge abutment. The present measurements were carried out in the course of the already described calibration truck-crossings. It was aspired to figure out, how the prevailing loading (predetermined wheel loads) act on the deck plate itself after some transverse redistribution of forces due to the deck plate's pavement, with an additionally manufactured zigzag-shear force retention. Comparable to Level I, a series of measurement should be generated by a few individual measurements. To cover their statistical randomness, the methodology introduced for Level I will have to be adopted for this level of analysis. 

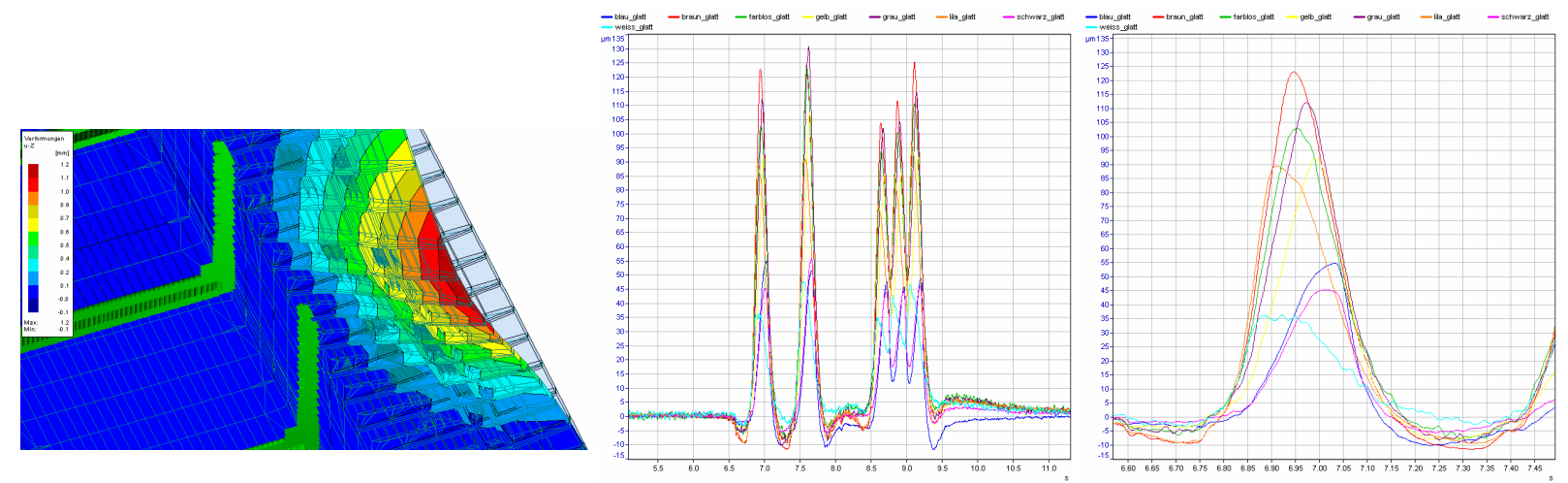

Fig. 23. The bay's deformation due to a truck passage with five axles

In Figure 23 (right) the bay's deformation due to a truck passage with 5 axles is visualised. The total displacement of the bay was recognised to be in the range of 50 and $130 \mu \mathrm{m}$ (Figure 23, right). A zoom into the obtained curves reveals very clearly, that the right bay - directly stressed by tire impact - was chosen. A comparison of the pattern's gradients shows the expected reaction. Those sensors, directly influenced in the beginning of the truck passage, respond with a gradient with a sudden, distinctive increase and a reduced decrease after the impact, while those transducers at the measurement grid's end react the other way round. The verification of plausibility can again be performed by FE-analysis, which confirmed this measured range (approximately $100 \mu \mathrm{m}$ ) for one single bay under explicit truck tire loading. Moreover it is to be emphasized, that the same range of deformation is achieved for a overhanging area of more than half a meter within the $7,50 \mathrm{~m}$ long cantilever, stressed by an unloaded truck with 8,7 tons (Figure 23, left).

The preliminary idea how to utilize the obtained data for stress analysis is to implement the measurement grid's information (18.05.2005 14:00 - 19.05.2005 01:00) into a forced vibration load case and pre-determine the hot spot areas to be analysed. Finite Element Analysis - in connection with that forced vibration load case - automatically leads to a time history pattern in terms of stresses which will have to be processed via Rainflow-couting and further superposition considerations due to existing sources of influence from Level I \& Level II.

\section{Remaining Service Lifetime by Means of Existing Traffic Data}

The assessment of an analysed detail is always carried out with a Damage Matrix calculated for the measuring time of a whole year. This matrix includes the so-called 'Damage-per year effect'. The detailed knowledge about the progression of the prevailing traffic from the very beginning up to these days and the implementation of published future trend studies about the next ten years can be used for fatigue analysis. Figure 24 shows the increase of the freight traffic volume at the Europabrücke. According to [1], traffic volume in 2004 increased to an amount of 406 $\%$ in relation to 1964 and is expected to grow 2.9\% per year until 2015 [1]. To derive every considered year's Damage Matrix affected by the variation of traffic volume, fatigue analysis approximately demands a uniform adaptation of the number of occurrences for all elements of the derived Rainflow Matrix before already discussed extrapolation techniques of the measured impact for the whole lifetime are applied. 


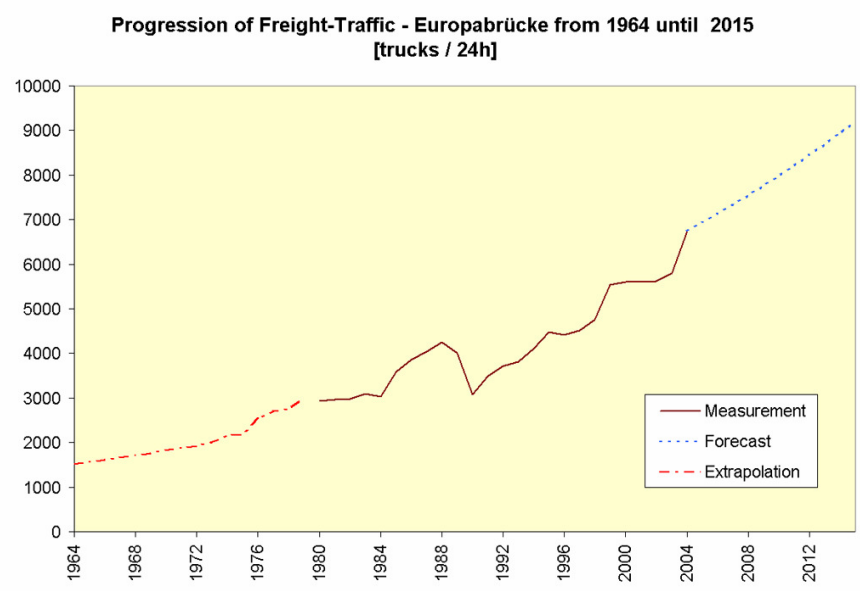

Fig.24. Quantitative development of traffic volume at the Europabrücke from 1964 until 2015 [motor vehicles/day]

Figure 25 shows the increase of the effective amount of transported goods compared to a calculated notional tonnage per truck. The calculations showed that this truck weight in 2004 increased to an amount of $506 \%$ in relation to 1964 and is assumed to have already reached a maximum [1]. This means, that a further increase of transported goods is likely to be a consequence of the still growing traffic volume. An adaptation for fatigue analysis due to the variation of the notional truck-weight is realised by re-scaling the Rainflow Matrice's subdivision.

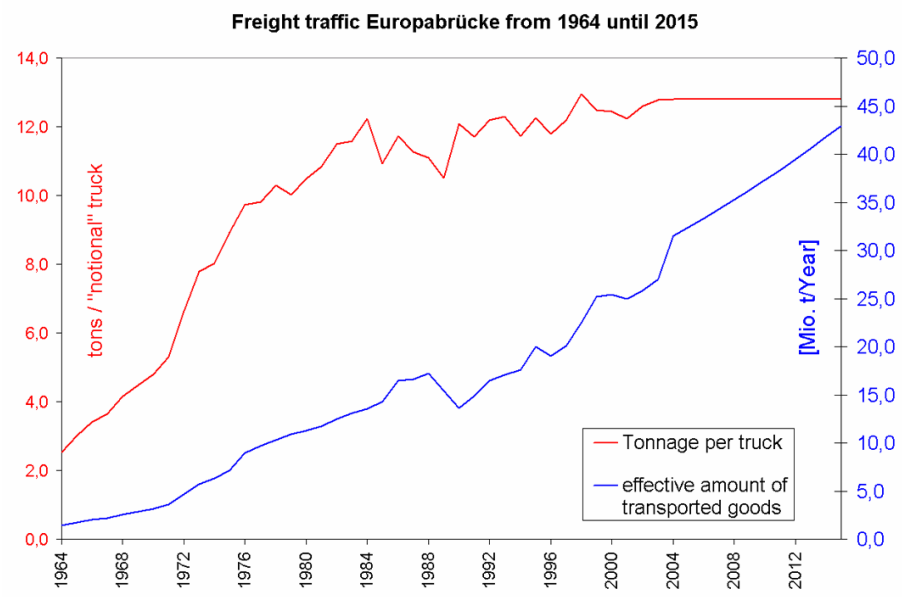

Fig. 25. Trend of the total freight traffic tonnages on the Brenner route compared to the cargo per truck

\section{Conclusions and Future Work}

The present contribution explicitly deals with measurement data and the procedures, how they were made available and conditioned. Thus the main goal, the substitution of the standard's premises - referring to loading - has been reached in a quite innovative manner to determine the consumption of the structure's overall-capacity per year by means of a three level approach.

Up to now the application of Palgrem-Miner's damage accumulation theory for the research of the consequences of randomly induced traffic loading based on a permanent measuring system has been shown. The latter was stepwise developed 
and modified. From July 2004 its present configuration fulfils all general requirements. In this respect the measurement over one whole calendar year has still not been completed.

The accumulation of all calculated Damage Matrices from the very beginning of the bridge's existence up to now leads to the remaining capacity of loading cycles for the analyzed detail. As a superior conclusion in addition to a quantitative estimation of the service-lifetime another key figure FR (Fatigue Relevance) is also derived (eq. (11)). It separates remaining, fatigue relevant loading cycles $\boldsymbol{n}_{i}$ (registered by sensors and taken from the Damage Matrix) from the randomly occurring traffic (ADTV = average daily traffic volume). It is obvious that the investigation's results are going to be improved by progressive stages; the longer the observation period lasts.

$$
F R=\frac{\sum n_{i}}{A D T V}
$$

The fatigue process can be subdivided into two phases; initiation (=development and early growth) and propagation (=growth of a crack to failure). Up to now the Stress Life Approach ('crack-initiation' with service life to form "small cracks") dealing with structural (Hot Spot) Wöhler-Curves given for $N \geq 10^{4}$ has been discussed, as it is best suited for truck traffic - causing High Cycle Fatigue. It does not distinguish between initiation and propagation phases, but deals with the total lifetime. Linear elastic material behaviour can be assumed, as the structural hot spot stress range should not exceed twice the yield strength of the material [3].

\section{Acknowledgments}

The authors would like to express their gratitude to the colleagues at ASAG (Alpenstrassen - Aktiengesellschaft) for their cooperativeness and the opportunity for this research work. 


\section{References}

[1] R. Veit, H. Wenzel, J. Fink., "Measurement data based lifetime-estimation of the Europabrücke due to traffic loading - a three level approach", Proceedings of the 58th International Conference of International Institute of Welding, Prague, Czech Republic (2005).

[2] Haibach E., "Betriebsfestigkeit - Verfahren und Daten zur Bauteilberechnung", 2, VDIVerlag, Düsseldorf (2002).

[3] Hobbacher A., "Recommendations for fatigue design of welded joints and components", International Institute of Welding, doc. XIII-1965-03/XV1127-03, Paris, France (2003).

[4] ESDEP - European Steel Design Education Program: WG12 Fatigue, Lecture Notes, Katholieke Universiteit Leuven

[5] Wenzel H., Pichler D., "Ambient Vibration Monitoring", J. Wiley and Sons Ltd, Chichester - England, 2005, ISBN 0470024305

[6] Spaethe G., "Die Sicherheit tragender Baukonstruktionen“, 2, Springer-Verlag, Wien New York (1992)

[7] Bronstein I., Semendjajew K.A., "Taschenbuch der Mathematik“, 5, Harri DeutschVerlag, Frankfurt am Main (2001)

[8] Dreßler K., Gründer B., Hack M., Köttgen V.B., "Extrapolation of Rainflow Matrices", SAE Technical Paper No. 960569 (1996) 